\title{
QGSJET-III model: physics and preliminary results
}

\author{
Sergey Ostapchenko ${ }^{1,2, \star}$ \\ ${ }^{1}$ Frankfurt Institute for Advanced Studies (FIAS), 60438 Frankfurt am Main, Germany \\ ${ }^{2}$ D.V. Skobeltsyn Institute of Nuclear Physics, Moscow State University, 119992 Moscow, Russia
}

\begin{abstract}
We discuss in some detail the physics content of the new model, QGSJET-III-01, focusing on major problems related to the treatment of semihard processes in the very high energy limit. A special attention has been payed to the main improvement, compared to the QGSJET-II model, which is related to a phenomenological treatment of leading power corrections corresponding to final parton rescattering off soft gluons. In particular, this allowed us to use a twice smaller separation scale between the soft and hard parton physics, compared to the previous model version, QGSJET-II-04. Preliminary results obtained with the new model are also presented.
\end{abstract}

\section{Introduction}

Modeling of high energy hadronic interactions is of considerable importance for experimental studies in the high energy cosmic ray field. Since one traditionally infers the information on the properties of primary cosmic ray particles from measured characteristics of nuclear-electromagnetic cascades induced by them in the atmosphere, the success of experimental studies depends on the validity of hadronic interaction models used in the analysis.

Since two dozens of years the QGSJET model [1-3] and its successor, QGSJET-II [4-7], have been broadly used in the high energy cosmic ray field. Here we are going to describe the underlying physics approaches, focusing on major problems related to the treatment of semihard processes in the very high energy limit, and discussing in some detail the most recent developments which formed the basis for the new model version, QGSJET-III.

The driving idea behind the QGSJET model has been a unified approach to the treatment of both nonperturbative soft and perturbative hard processes in high energy interactions of hadrons and nuclei $[2,3,8,9]$. However, including a treatment of hard processes in a Monte Carlo generator is like opening a Pandora box: numerous self-consistency problems emerge faster than the respective solutions. Among the most important ones is a very rapid energy-rise of the interaction cross sections and of the yields of produced particles, which is closely related to a steep increase of parton momentum distribution functions (PDFs) in the limit of small parton momenta. While that was not an issue for the original QGSJET, which was based on flat pre-HERA PDFs, developing a selfconsistent formalism based on realistic PDFs proved to be a very complicated task. The key step in this direction has been a treatment of nonlinear interaction effects, based

\footnotetext{
^e-mail: ostapchenko@fias.uni-frankfurt.de
}

on all-order resummation of the underlying, so-called enhanced, Pomeron diagrams [10-12], which has been the major feature of QGSJET-II. Here we are going to discuss the implementation of hopefully the last but so far missing important ingredient of the approach, which is related to the treatment of higher twist corrections to hard parton processes.

\section{Jet production and the energy rise of the total $p p$ cross section}

It has been realized long ago that interactions of hadrons at very high energies are dominated by production of hadronic jets of moderately large transverse momenta $p_{\mathrm{t}}$ [13]. The collinear factorization of the perturbative quantum chromodynamics (pQCD) $[14,15]$ allows one to calculate the energy-dependence of the inclusive jet production cross section $\sigma_{p p}^{\text {jet }}\left(s, p_{t}^{\text {cut }}\right)$, for $p_{\mathrm{t}}$ larger than some chosen cutoff $p_{t}^{\text {cut }}$, as the latter is expressed via the relatively well-known PDFs $f_{I / p}\left(x, Q^{2}\right)$ :

$$
\begin{array}{r}
\sigma_{p p}^{\mathrm{jet}}\left(s, p_{\mathrm{t}}^{\mathrm{cut}}\right)=\sum_{I, J=q, \bar{q}, g} \int_{p_{\mathrm{t}}>p_{\mathrm{t}}^{\text {cut }}} d p_{t}^{2} \int d x^{+} d x^{-} \\
\times f_{I / p}\left(x^{+}, M_{\mathrm{F}}^{2}\right) f_{J / p}\left(x^{-}, M_{\mathrm{F}}^{2}\right) \frac{d \sigma_{I J}^{2} \rightarrow^{2}\left(x^{+} x^{-} s, p_{t}^{2}, M_{\mathrm{F}}^{2}\right)}{d p_{t}^{2}} .
\end{array}
$$

Here $s$ is the center-of-mass (c.m.) energy squared for the scattering, $x^{ \pm}$- the light cone (LC) momentum fractions of the partons $I$ and $J$ [(anti-)quarks or gluons], taking part in the hard process, $d \sigma_{I J}^{2 \rightarrow 2} / d p_{t}^{2}$ is the parton scatter cross section, and $M_{\mathrm{F}}^{2}$ - a chosen factorization scale. The energy rise of $\sigma_{p p}^{\text {jet }}$ is thus related to the low $x$ behavior of the PDFs $f_{I / p}\left(x, Q^{2}\right)$, which is driven, in turn, by the increase of the phase space for parton evolution. Describing the latter by the Dokshitzer-Gribov-Lipatov-Altarelli-Parisi (DGLAP) 
equations, we have approximately:

$$
\sigma_{p p}^{\text {jet }}\left(s, Q_{0}\right) \propto \frac{1}{Q_{0}^{2}} s^{\Delta_{\text {eff }}}, \quad \Delta_{\text {eff }} \sim 0.3 .
$$

Here we immediately recognize two problems. First, the inclusive jet production cross section depends strongly on the chosen $p_{t}$-cutoff $p_{t}^{\text {cut }}$. Secondly and more importantly, $\sigma_{p p}^{\text {jet }}$ rises asymptotically much quicker than the total cross section $\sigma_{p p}^{\mathrm{tot}}(s)$, as the latter can not rise quicker than $\propto \ln ^{2} s$. Thus, at sufficiently high energies one deals with multiple jet production per inelastic collision, which is usually referred to as multiparton interactions (MPIs) [16].

\section{MPIs: eikonal approach}

Generally, the usual PDFs are insufficient to treat multiparton interactions. Rather, the corresponding formalism involves the so-called generalized multiparton distributions ( $\left.{ }^{n} \mathrm{GPDs}\right) F_{I_{1}, \ldots I_{n}}^{(n)}\left(x_{1}, \ldots x_{n}, \vec{b}_{1}, \ldots \vec{b}_{n}, Q_{1}^{2}, \ldots Q_{n}^{2}\right)$ which describe a simultaneous distribution of $n$ partons with respect to their light cone momentum fractions $x_{i}$ and transverse coordinates $\vec{b}_{i}$, when probed at the corresponding virtuality scales $Q_{i}^{2}$ [17-19]. For example, the expression for double parton scattering (DPS) cross section contains ${ }^{2}$ GPDs $F_{I_{1}, I_{2}}^{(2)}$ for the projectile and target protons:

$$
\begin{array}{r}
\sigma_{p p}^{4 j \mathrm{jet}(\mathrm{DPS})}\left(s, p_{\mathrm{t}}^{\mathrm{cut}}\right)=\frac{1}{2} \int d x_{1}^{+} d x_{2}^{+} d x_{1}^{-} d x_{2}^{-} \\
\times \int_{p_{\mathrm{t}_{1}}, p_{\mathrm{t}_{2}}>p_{\mathrm{t}}^{\text {cut }}} d p_{\mathrm{t}_{1}}^{2} d p_{\mathrm{t}_{2}}^{2} \sum_{I_{1}, I_{2}, J_{1}, J_{2}} \frac{d \sigma_{I_{1} J_{1}}^{2 \rightarrow}}{d p_{\mathrm{t}_{1}}^{2}} \frac{d \sigma_{I_{2} J_{2}}^{2 \rightarrow 2}}{d p_{\mathrm{t}_{2}}^{2}} \\
\times \int d^{2} \Delta b F_{I_{1} I_{2}}^{(2)}\left(x_{1}^{+}, x_{2}^{+}, M_{\mathrm{F}_{1}}^{2}, M_{\mathrm{F}_{2}}^{2}, \Delta b\right) \\
\times F_{J_{1} J_{2}}^{(2)}\left(x_{1}^{-}, x_{2}^{-}, M_{\mathrm{F}_{1}}^{2}, M_{\mathrm{F}_{2}}^{2}, \Delta b\right) .
\end{array}
$$

Since there exists a very scarce experimental information on multiparton GPDs, a standard simplifying assumption is to neglect multiparton correlations, in which case $n$-parton GPDs factorize into a product of $n$ independent single-parton GPDs:

$$
F_{I_{1} \ldots I_{n}}^{(n)}\left(x_{1}, \ldots x_{n}, \vec{b}_{1}, \ldots \vec{b}_{n}, Q_{1}^{2}, \ldots Q_{n}^{2}\right)=\prod_{i=1}^{n} G_{I_{i}}\left(x_{i}, \vec{b}_{i}, Q_{i}^{2}\right)
$$

the latter being subject to a constraint

$$
\int d^{2} b G_{I}\left(x, \vec{b}, Q^{2}\right)=f_{I / p}\left(x, Q^{2}\right) .
$$

In such a case, Eq. (3) simplifies to

$$
\sigma_{p p}^{4 \mathrm{jet}(\mathrm{DPS})}\left(s, p_{\mathrm{t}}^{\mathrm{cut}}\right)=\frac{1}{2} \int d^{2} b\left[2 \chi_{p p}^{\mathrm{jet}}\left(s, b, p_{\mathrm{t}}^{\mathrm{cut}}\right)\right]^{2}
$$

being expressed via the so-called jet production eikonal $\chi_{p p}^{\text {jet }}$, with [c.f. Eq. (1)]

$$
\begin{array}{r}
\chi_{p p}^{\text {jet }}\left(s, b, p_{\mathrm{t}}^{\text {cut }}\right)=\frac{1}{2} \sum_{I, J} \int_{p_{\mathrm{t}}>p_{\mathrm{t}}^{\text {cut }}} d p_{t}^{2} \int d x^{+} d x^{-} \\
\times \int d^{2} \Delta b G_{I}\left(x^{+}, \vec{b}, M_{\mathrm{F}}^{2}\right) G_{J}\left(x^{-}, \vec{b}+\overrightarrow{\Delta b}, M_{\mathrm{F}}^{2}\right) \\
\times \frac{d \sigma_{I J}^{2 \rightarrow 2}\left(x^{+} x^{-} s, p_{t}^{2}, M_{\mathrm{F}}^{2}\right)}{d p_{t}^{2}} .
\end{array}
$$

More generally, for $n$-parton scattering one has

$$
\sigma_{p p}^{\mathrm{jet}(n)}\left(s, p_{\mathrm{t}}^{\mathrm{cut}}\right)=\frac{1}{n !} \int d^{2} b\left[2 \chi_{p p}^{\mathrm{jet}}\left(s, b, p_{\mathrm{t}}^{\mathrm{cut}}\right)\right]^{n} .
$$

In order to see the effect of multiparton interactions on the total $p p$ cross section, we have to take into account that multiple parton scattering generates a number of other processes, in addition to multiple jet production, as exemplified in Fig. 1 for the particular case of double parton scattering. The first (cut) diagram in Fig. 1 corresponds to the partial contribution to $\sigma_{p p}^{\text {tot }}$ from the abovediscussed double dijet production process; there are two real parton cascades giving rise to secondary hadron production. The second graph in Fig. 1 describes a (negative) screening correction to single dijet production process; in addition to the real parton cascade giving rise to hadron production, there is a virtual cascade responsible for an elastic rescattering process. Finally, the last diagram in Fig. 1 corresponds to a (quasi)elastic scattering: both parton cascades are virtual ones, hence, they do not contribute to hadron production. Using the AbramovskiiGribov-Kancheli (AGK) cutting rules [20], one obtains the relation between the relative contributions of the three graphs in Fig. 1 as (+2):(-4):(+1). Consequently, the summary contribution of double parton scattering to $\sigma_{p p}^{\text {tot }}$ can be expressed via the double dijet production cross section as

$$
\Delta^{(2)} \sigma_{p p}^{\mathrm{tot}}=-\frac{1}{2} \sigma_{p p}^{4 \mathrm{jet}(\mathrm{DPS})} .
$$

Performing a similar analysis for $n$-parton scattering, $n>2$, one arrives to the well-known "minijet" ansatz for the total $p p$ cross section: ${ }^{1}$

$$
\sigma_{p p}^{\mathrm{tot}}\left(s, p_{\mathrm{t}}^{\mathrm{cut}}\right)=2 \int d^{2} b\left[1-e^{-\chi_{p p}^{\mathrm{jet}}\left(s, b, p_{\mathrm{t}}^{\mathrm{cut}}\right)}\right] .
$$

It is important to notice that the above-discussed multiple scattering processes have no impact on the inclusive jet production cross section $\sigma_{p p}^{\mathrm{jet}}$. Indeed, summing up the partial contributions of the three graphs in Fig. 1, we have

$$
\Delta^{(2)} \sigma_{p p}^{\mathrm{jet}}=2 \sigma_{p p}^{4 \mathrm{jet}(\mathrm{DPS})}[(1) * 2+(-2) * 1+(1 / 2) * 0]=0,
$$

where we take into account that the 1st graph contributes twice (two dijets produced) while the last diagram gives a zero contribution. This is known as the AGK-cancellations [20], which hold also for an arbitrary $n$-parton scattering. As a result, $\sigma_{p p}^{\text {jet }}$ remains defined by Eq. (1) and the collinear pQCD factorization holds.

\footnotetext{
${ }^{1}$ For the sake of simplicity, we don't discuss explicitly the contribution of soft $\left(p_{\mathrm{t}}<p_{\mathrm{t}}^{\text {cut }}\right)$ particle production to $\sigma_{p p}^{\mathrm{tot}}$.
} 


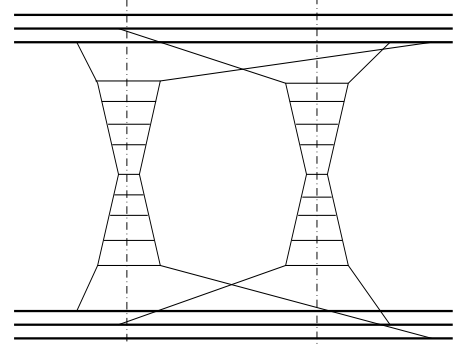

$(+2)$

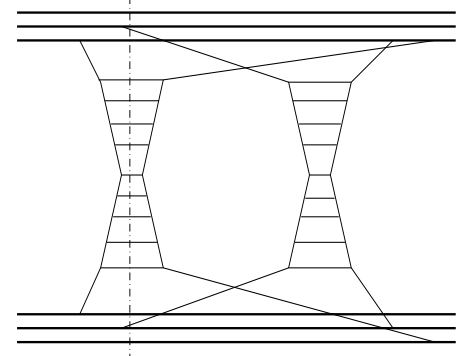

$(-4)$

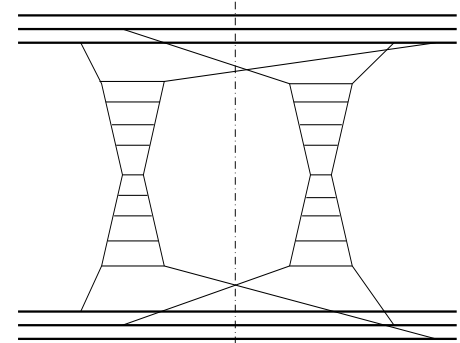

$(+1)$

Figure 1. Schematic diagrams for different processes generated by double parton scattering in proton-proton collisions: double dijet production (left), screening correction to single dijet production (middle), and (quasi)elastic scattering (right); the vertical dashed-dotted lines indicate the position of the cut plane. Relative weights of the corresponding contributions are shown below the graphs.

\section{MPIs: role of color fluctuations}

At the first sight, the eikonalization procedure described in Section 3 allows one to "marry" the quick energy rise of $\sigma_{p p}^{\text {jet }}$ with a relatively slow increase of $\sigma_{p p}^{\text {tot }}$. Since the total $p p$ cross section depends strongly on the effective area occupied by partons in the transverse plane [c.f. Eqs. (7) and (10)], one seems to have a possibility to properly tune model predictions for $\sigma_{p p}^{\text {tot }}$ by adjusting the energy-dependence for spacial parton distributions. Indeed, choosing a more dilute parton distribution over a larger area at a given collision energy, as depicted in Fig. 2(left), one obtains a larger $\sigma_{p p}^{\text {tot }}$ and a smaller number of
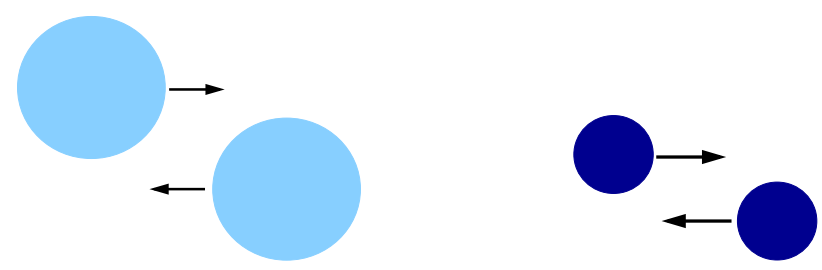

Figure 2. Schematic view of proton-proton collisions for a broader (left) or narrower (right) proton transverse profile.

jets produced per inelastic event. On the contrary, having a denser parton cloud occupying a smaller area, as in Fig. 2(right), gives rise to a smaller $\sigma_{p p}^{\text {tot }}$ but to a higher jet production rate. In reality, there is a little freedom here since the effective proton size is strongly constrained by experimental measurements of the elastic scattering slope $B_{p p}^{\mathrm{el}}(s)$, which is proportional to the average impact parameter squared for $p p$ collision, $B_{p p}^{\mathrm{el}} \propto\left\langle b^{2}\right\rangle$, notably, by the respective data of the TOTEM and ATLAS experiments [21-25] at the Large Hadron Collider (LHC). As demonstrated in Ref. [26], using a realistic spacial parton distribution in the proton results in a too fast energy-rise of $\sigma_{p p}^{\text {tot }}$, compared to experimental data.

Potentially, an additional freedom comes from the treatment of color fluctuations in the proton [27]. We may consider the proton to be represented by a superposition of a number of Fock states characterized by different sizes and parton densities, as depicted in Fig. 3, i.e.

$$
|p\rangle=\sum_{i} \sqrt{C_{i}}|i\rangle
$$

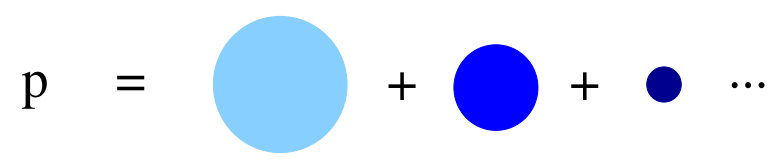

Figure 3. Schematic view for the decomposition of the proton wave function in terms of parton Fock states of different transverse sizes.

with $C_{i}$ being the respective partial weights, $\sum_{i} C_{i}=1$. Then Eq. (10) changes to

$$
\sigma_{p p}^{\mathrm{tot}}\left(s, p_{\mathrm{t}}^{\mathrm{cut}}\right)=2 \sum_{i, j} C_{i} C_{j} \int d^{2} b\left[1-e^{-\chi_{i j}^{\mathrm{jet}}\left(s, b, p_{\mathrm{t}}^{\mathrm{cut}}\right)}\right],
$$

where the eikonal $\chi_{i j}^{\text {jet }}$ describes jet production for the case when the projectile and target protons are represented by the states $|i\rangle$ and $|j\rangle$, respectively. $\chi_{i j}^{\text {jet }}$ is defined by Eq. (7), with the GPDs $G_{I}\left(x, \vec{b}, Q^{2}\right)$ being replaced by the partial ones $G_{I /(i)}\left(x, \vec{b}, Q^{2}\right)$, for the state $|i\rangle$ of interest, with

$$
\sum_{i} C_{i} G_{I /(i)}\left(x, \vec{b}, Q^{2}\right)=G_{I}\left(x, \vec{b}, Q^{2}\right) .
$$

Thus, allowing for a larger dispersion between the properties of Fock states $|i\rangle$, one may reduce $\sigma_{p p}^{\text {tot }}$, for a given $\sigma_{p p}^{\text {jet }}$. Indeed, the main contribution to the total $p p$ cross section comes from the largest size states of the projectile and target protons (c.f. Figs. 2 and 3). On the other hand, the above-discussed decomposition of the proton wave function has no impact on $\sigma_{p p}^{\text {jet }}\left(s, p_{t}^{\text {cut }}\right)$ : due to the constraints (5) and (14), it remains defined by Eq. (1).

However, choosing a larger dispersion between the properties of proton's Fock states, one obtains a higher cross section for (single plus double) low mass diffraction (LMD):

$$
\begin{array}{r}
\sigma_{p p}^{\mathrm{LMD}}\left(s, p_{\mathrm{t}}^{\mathrm{cut}}\right)=\int d^{2} b\left\{\left[\sum_{i, j} C_{i} C_{j}\left(1-e^{-\chi_{i j}^{\mathrm{jet}}\left(s, b, p_{\mathrm{t}}^{\mathrm{cut}}\right)}\right)^{2}\right]\right. \\
\left.-\left[\sum_{i, j} C_{i} C_{j}\left(1-e^{-\chi_{i j}^{\mathrm{jet}}\left(s, b, p_{\mathrm{t}}^{\mathrm{cut}}\right)}\right)\right]^{2}\right\} .
\end{array}
$$

On the other hand, $\sigma_{p p}^{\mathrm{LMD}}$ at the LHC energies is constrained by the data of the TOTEM experiment [28, 29]. 


\section{MPIs: role of multiparton correlations}

As is clear from the above discussion, for a given inclusive jet production cross section, $\sigma_{p p}^{\text {tot }}$ anticorrelates with the average jet production rate per inelastic event. Do we have any means to enhance the latter without changing the transverse profile of the proton? This turns to be possible indeed, if we reconsider the basic assumption of the minijet approach, Eq. (4), and take into account multiparton correlations [30]. The main problem with the uncorrelated parton picture is illustrated qualitatively in Fig. 4(left). Considering for the sake of simplicity a top-hat
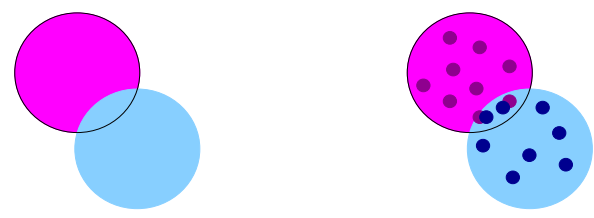

Figure 4. Schematic view for the overlap between parton clouds of the colliding protons for a uniform parton distribution in the proton disk (left) and for a clumpy profile of the proton (right).

transverse profile for the proton, partons are uniformly distributed in the proton disk. In such a case, multiple jet production mostly takes place in relatively central $p p$ collisions characterized by small impact parameters, where one has a significant overlap of the projectile and target parton clouds. In turn, this results in a small average number of jets produced per inelastic event. As an alternative, we may assume that parton-parton correlations give rise to parton "clumps", as depicted in Fig. 4(right). In the latter case, multiple jet production is considerably enhanced in peripheral collisions, which thus enlarges also the average jet production rate per event.

A mechanism of that kind emerges in the QGSJET-II model $[5,6]$. Being designed for a combined treatment of soft and hard processes, the model describes parton evolution in the nonperturbative region of low parton virtualities, $\left|q^{2}\right|<Q_{0}^{2}, Q_{0}$ being some cutoff for pQCD being applicable, as an emission of a soft Pomeron [2, 3, 9], as illustrated in Fig. 5. In addition, in contrast to the tradi-

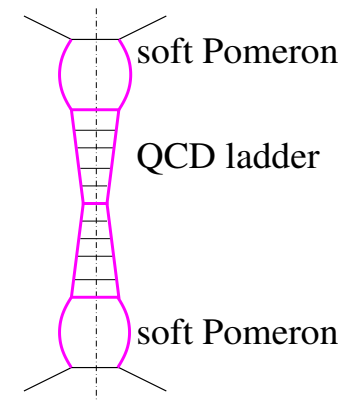

Figure 5. Schematic view of a hard scattering process: perturbative parton evolution is represented by the ladder; the "blobs" above and below the ladder correspond to nonperturbative parton cascades described as soft Pomeron emission.

tional eikonal treatment described in Section 3, one takes into consideration nonlinear effects related to interactions between parton cascades, which is treated as PomeronPomeron interactions [5, 6, 10-12]. Among other effects, this introduces special contributions to multiparton GPDs, which correspond to a "soft parton splitting" mechanism [31], as illustrated in Fig. 6 for the case of double dijet production. The two partons entering the two ladders

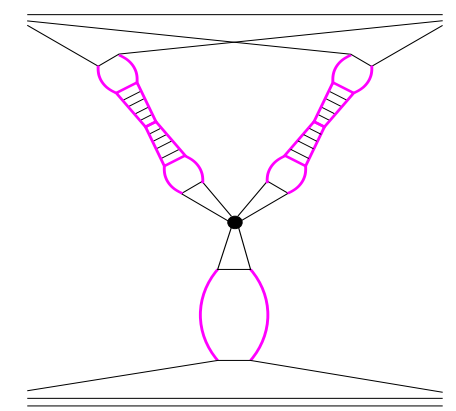

Figure 6. Schematic representation for the contribution of the "soft parton splitting" mechanism to double dijet production. Perturbative parton cascades are represented by the ladders and soft parton evolution - by the "blobs"; the triple-Pomeron interaction vertex is shown by the small black circle.

from below, i.e. the initial partons for the two perturbative $\left(\left|q^{2}\right|>Q_{0}^{2}\right)$ parton cascades, originate from two soft Pomerons describing nonperturbative parton evolution. In turn, those soft Pomerons emerge from a splitting of their parent soft Pomeron coupled to the target proton. Thus, we have the following picture: a soft parton cascade developing in the target proton, followed by a splitting of the last parton in this cascade into a pair of new soft partons which, in turn, initiate two separate soft cascades. Finally, each of those enters the perturbative $\left(\left|q^{2}\right|>Q_{0}^{2}\right)$ domain and gives rise to a dijet production. Because of the relatively small slope of the soft Pomeron, the two partons entering the perturbative evolution appear to be close-by in the transverse plane, i.e. we have effectively a parton clump.

In the above-described approach, one obtains a reasonable description of both total and elastic $p p$ cross sections, when using realistic PDFs [5, 6]. On the other hand, due to the AGK-cancellations, the scheme preserves the collinear QCD factorization, with $\sigma_{p p}^{\text {jet }}$ being defined by Eq. (1) [5].

\section{Energy-rise of $N_{p p}^{\mathrm{ch}}$ and higher twist corrections}

As stressed repeatedly in the previous Sections, a very important feature of the above-discussed approaches is that they preserve the collinear factorization result, Eq. (1), for inclusive jet production. Yet this may be a problem when applied to particle production. First, as already mentioned in Section 2, the inclusive jet rates, hence, also the multiplicity of produced hadrons, depend strongly on the chosen transverse momentum cutoff, see Eq. (2). Secondly, the corresponding energy-dependence predicted by pQCD evolution of parton densities, $\sigma_{p p}^{\text {jet }}\left(s, Q_{0}\right) \propto s^{\Delta_{\text {eff }}}$, is much steeper than the observed energy-rise of the number of 
charged hadrons produced in $p p$ collisions, $N_{p p}^{\mathrm{ch}}(s) \propto s^{0.13}$ [32].

In the QGSJET-II-04 model, a relatively high cutoff $Q_{0}^{2}=3 \mathrm{GeV}^{2}$ is employed as the border between the perturbative hard and nonperturbative soft parton evolution. Moreover, for the chosen factorization scale $M_{\mathrm{F}}^{2}=p_{\mathrm{t}}^{2} / 4$, this transforms into a pretty high cutoff $p_{\mathrm{t}}^{\text {cut }} \simeq 3.4 \mathrm{GeV}$ for the hardest parton scattering process. From general considerations, one may expect pQCD to be applicable down to much smaller values $p_{\mathrm{t}} \sim 1 \mathrm{GeV}$. Hence, an important perturbative mechanism seems to be missing.

Let us now remind ourselves that the collinear factorization of pQCD has been established at the leading twist level, i.e. neglecting the so-called higher twist (HT) corrections suppressed by additional powers of the hard scale $[14,15]$. It is thus natural to expect that these are HT effects which damp jet production for relatively small jet transverse momenta, while being of minor importance at high $p_{\mathrm{t}}$ (being suppressed by additional powers of $p_{\mathrm{t}}$ ).

While the theoretical treatment of higher twist corrections dates 40 years back [33-37], a rigorous implementation of the formalism in Monte Carlo generators seems hardly possible at the present stage. The corresponding contributions involve many unknown multiparton correlators and generally do not allow a probabilistic treatment. Consequently, we are going to apply a highly phenomenological approach involving a number of ad hoc assumptions.

First of all, we concentrate on the contributions corresponding to parton rescattering on soft gluons, i.e. ones characterized by vanishingly small LC momentum fractions, $x_{g} \sim 0$, with the gluon pair forming a color singlet. A generic diagram of the kind is shown in Fig. 7 for the

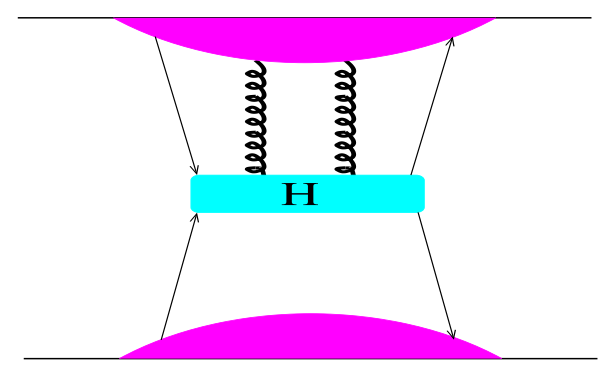

Figure 7. Generic diagram for higher twist corrections discussed in the text, for the case of quark-quark hard scattering.

case of quark-quark scattering. For a scattering on nuclear targets, such contributions have previously been shown to provide corrections to jet $p_{t}$-spectra, which are strongly enhanced by the size of the nucleus [38-40]. More specifically, we restrict ourselves with the diagrams describing final parton rescattering on a pair of virtual soft gluons, which have been shown to play the dominant role in the suppression of jet $p_{t}$-spectra in the low $x$ regime $[39,40]$. It is worth stressing that this is a strong ad hoc assumption since, unlike the case of a nuclear target, there is no formal justification for neglecting other potential HT contributions in the case of proton-proton interactions.
The corresponding expressions involve a quark-gluon correlation function

$$
\begin{array}{r}
T_{q g}\left(x_{q}, x_{g_{1}}=0, x_{g_{2}}=0\right)=\int \frac{d y_{q}^{-}}{4 \pi} \frac{d y_{g_{1}}^{-}}{2 \pi} \frac{d y_{g_{2}}^{-}}{2 \pi} \\
\times e^{i p^{+} x_{q} y_{q}^{-}}\left\langle p\left|\bar{\psi}(0) \gamma^{+} F_{+\alpha}\left(y_{g_{2}}^{-}\right) F_{\alpha}^{+}\left(y_{g_{1}}^{-}\right) \psi\left(y_{q}^{-}\right)\right| p\right\rangle \\
\times \Theta\left(y_{g_{2}}^{-}\right) \Theta\left(y_{g_{1}}^{-}-y_{q}^{-}\right)
\end{array}
$$

in the case of (anti-)quark-(anti-)quark or (anti-)quarkgluon scattering and a similar gluon-gluon correlator $T_{g g}$ for gluon-(anti-)quark or gluon-gluon scattering [39]. A closer look at Eq. (16) reveals that it formally coincides, up to a factor, with the quark-gluon ${ }^{2} \mathrm{GPD}$ $F_{q g}^{(2)}\left(x_{q}, x_{g}, Q_{q}^{2}, Q_{g}^{2}, \Delta b\right)$ multiplied by the gluon LC momentum fraction $x_{g}$, in the limit $x_{g} \rightarrow 0$ and for zero transverse separation between the two partons, $\Delta b=0$. This motivated us to employ a probabilistic treatment for $T_{q g}$ and $T_{g g}$ interpreting them as $x_{g} F_{q g}^{(2)}$ and $x_{g} F_{g g}^{(2)}$, respectively, and describing the latter by soft Pomeron asymptotics. We include also relevant absorptive corrections due to intermediate soft parton rescattering, described by enhanced Pomeron diagrams.

Here we have to make additional assumptions concerning the relevant virtuality scales $Q_{q}^{2}, Q_{g}^{2}$ and the gluon momentum fraction $x_{g}$. While the natural definition for $Q_{q}^{2}$ is the factorization scale $M_{\mathrm{F}}^{2}$ for the hard process, one usually considers soft gluons to be purely nonperturbative ones, with $Q_{g}^{2} \sim \lambda_{\mathrm{QCD}}^{2}$. We rather prefer to set $Q_{g}^{2}$ equal to our separation scale $Q_{0}^{2}$. Finally we take into consideration that the soft gluons have a finite virtuality, $\left|q_{g}^{2}\right| \sim\left\langle p_{\mathrm{t}}^{2}\right\rangle \sim x_{g} x_{g}^{-} s$, with $x_{g}^{-}$being the LC minus fraction for the gluon. Since our soft gluons belong to the projectile proton (for the diagram in Fig. 7), $x_{g}^{-}$should be much smaller than the LC minus fraction for the target quark participating in the hard process:

$$
x_{g}^{-} \sim \frac{\left\langle p_{\mathrm{t}}^{2}\right\rangle}{x_{g} s} \ll x_{q^{\prime}}^{-} .
$$

Since we expect a rather weak $x_{g}$-dependence for $x_{g} F_{q g}^{(2)}$ and $x_{g} F_{g g}^{(2)}$ in the small $x_{g}$ limit, we thus set

$$
x_{g}=\frac{Q_{0}^{2}}{x_{q^{\prime}}^{-} s} .
$$

As the above-described treatment contains a number of brute force assumptions and uncertainties, we introduce a new adjustable parameter to the model, which controls the magnitude of such contributions.

\section{QGSJET-III: preliminary results}

Including the mechanism described in Section 6, we were able to reach a consistent description of experimental data on total and elastic $p p$ cross sections and on particle production, using a twice smaller separation scale between the soft and hard parton dynamics, $Q_{0}^{2}=1.5 \mathrm{GeV}^{2}\left(p_{\mathrm{t}}^{\text {cut }} \simeq 2.4\right.$ $\mathrm{GeV}$ ), compared to the one in QGSJET-II-04. It is noteworthy that we also used a $30 \%$ larger value for the triplePomeron coupling, which gives rise to a considerably 
larger rate of high mass diffraction, in order to improve the agreement with diffraction studies by ATLAS and CMS $[41,42]$. On the other hand, the rate of low mass diffraction has been substantially reduced, to approach the respective TOTEM results [28].

The relative importance of the above-described different mechanisms for the cross section predictions is illustrated in Fig. 8, where we plot the results of the prelim-

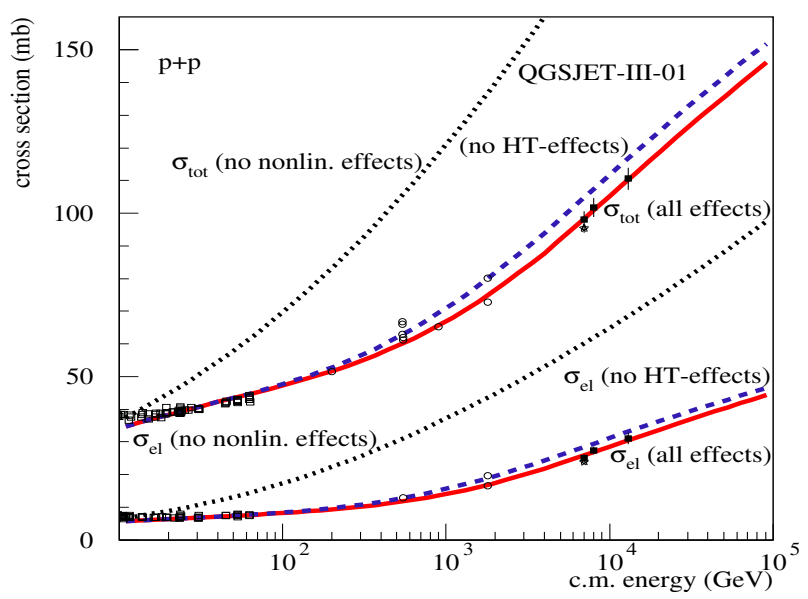

Figure 8. Energy-dependence of the total and elastic protonproton cross sections, as calculated using QGSJET-III-01 (solid lines), omitting the HT corrections (dashed lines), or neglecting all nonlinear effects (dotted lines). The experimental data are from Refs. [22-25, 43].

inary version of the new model, QGSJET-III-01, by solid lines. Omitting the HT corrections, we obtained the results shown by the dashed lines, while the dotted lines were obtained neglecting also the nonlinear interaction effects due to enhanced Pomeron diagrams. A similar comparison for the pseudorapidity density of produced charged hadrons and for the respective transverse momentum spectra for $\sqrt{s}=0.9$ and $13 \mathrm{TeV}$ is shown in Fig. 9. As one can see from the Figures, the main effect on both the cross sections and particle production comes from the treatment of nonlinear effects related to Pomeron-Pomeron interactions. For the current model parameter tune, higher twist contributions provide a smaller but important correction. For example, the overall reduction of the jet production rate for $p_{\mathrm{t}}>p_{\mathrm{t}}^{\text {cut }}$ amounts to $25 \%$, the respective effect on the hadron $p_{\mathrm{t}}$-spectra fading away with increasing $p_{\mathrm{t}}$ [see Fig. 9(right)].

Such a subdominant role of the HT effects in the current model implementation is partly related to the fact that we restrict ourselves with the simplest (so-called leading power) corrections shown schematically in Fig. 7. As a consequence, we had to put the respective normalization relatively low, in order to avoid substantial contributions from higher order power corrections, which involve additional soft gluons coupled to the hard process. In contrast, the treatment of nonlinear effects related to PomeronPomeron interactions is based on all-order resummation of the corresponding enhanced diagrams [10-12]. In principle, taking into consideration higher power corrections, in the spirit of the approach of Refs. [39, 40], may change somewhat this picture.

Concerning the predictions of the new model for extensive air shower (EAS) characteristics, it is probably a premature to discuss those in detail, first of all, because further development of the approach may have a noticeable impact on the results. Additionally, since the new model has been tuned to more or less the same data set as QGSJET-II-04, with the exception of the data on inelastic diffraction, one should not expect substantial differences for the predicted EAS properties. As an example, we show in Fig. 10 the calculated energy-dependence of the shower maximum depth $X_{\max }$. The obtained $\simeq 5 \mathrm{~g} / \mathrm{cm}^{2}$ shift between the results of the two models is driven by the above-discussed calibration of QGSJET-III-01 to the LHC diffractive data.

\section{Outlook}

We discussed in some detail the physics content of the new model, QGSJET-III, focusing on major problems related to the treatment of semihard processes in the very high energy limit. In particular, a special attention has been payed to the main improvement, compared to QGSJET-II, which is related to a phenomenological treatment of leading power corrections corresponding to final parton rescattering off soft gluons. It has been demonstrated that this new mechanism has a subdominant but important effect on the model predictions for the interaction cross sections and particle production. Yet a further development of the approach, aiming on including also higher power corrections, seems desirable. On the other hand, an independent calibration of the normalization of the respective contributions, e.g. based on experimental data on hadron transverse spectra in the range of moderately small $p_{\mathrm{t}}$ [47], would enhance the reliability of the model results.

Overall, the developed approach is a highly phenomenological one, hence, its merit may only come from a successful description of various experimental data. Nevertheless, even the current implementation is a substantial step forward compared to the standard recipee used in most of the present Monte Carlo generators: ad hoc parametrized energy-dependent $p_{\mathrm{t}}$-cutoff for hard processes, which, in fact, breaks the link between the measured PDFs and the cross section predictions. In contrast, we have a dynamical treatment of the damping of jet production in the range of moderately small $p_{\mathrm{t}}$, using a single new adjustable parameter.

Concerning potential applications in the high energy cosmic ray field, additional, more technical, improvements of the model may also be necessary, notably, in relation to forward hadron production. While the importance of the possible $\pi$-meson exchange dominance for the forward $\rho$-meson production in pion-proton and pion-nucleus collisions has already been stressed in Ref. [7], there are currently strong experimental indications on the dominant role of the pion exchange for forward neutron production in $p p$ interactions $[48,49]$. Hence, a self-consistent implementation of the treatment of Reggeon-Reggeon-Pomeron 

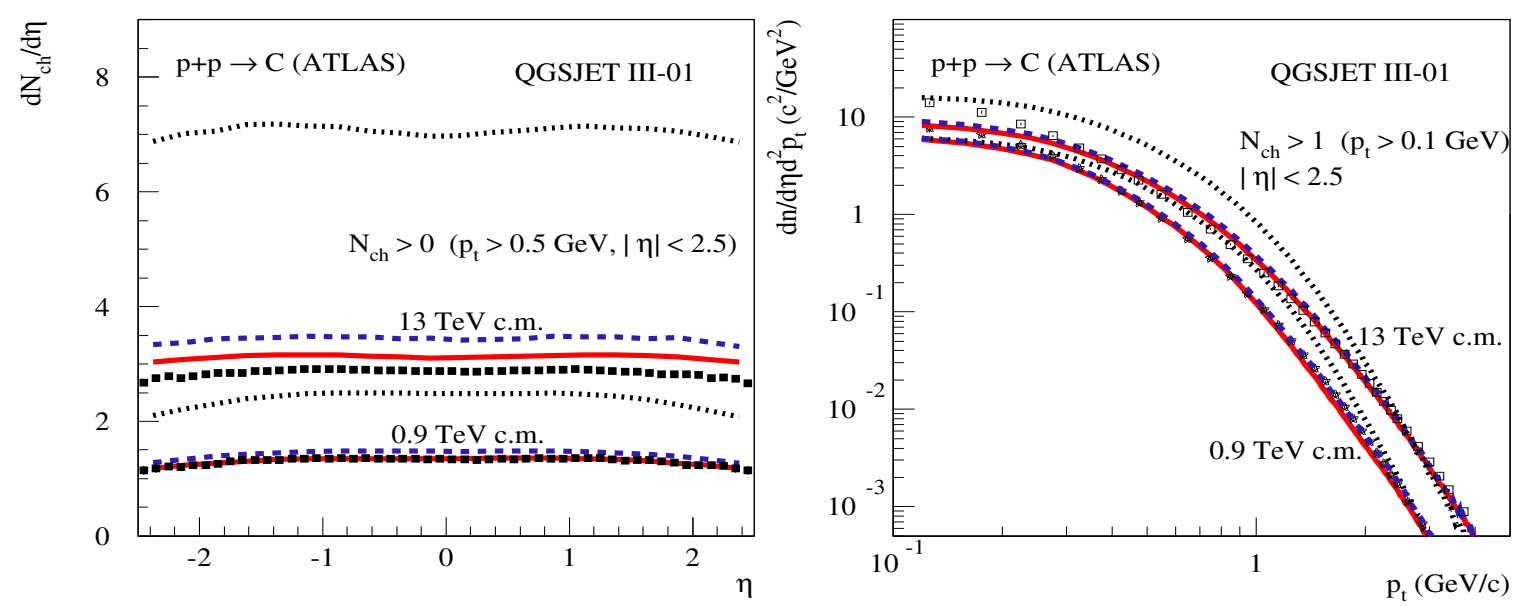

Figure 9. Calculated pseudorapidity density of produced charged hadrons (left) and transverse momentum spectra of charged hadrons (right) for $\sqrt{s}=0.9$ and $13 \mathrm{TeV}$, compared to the data of the ATLAS experiment [44-46]. The meaning of the lines is the same as in Fig. 8 .

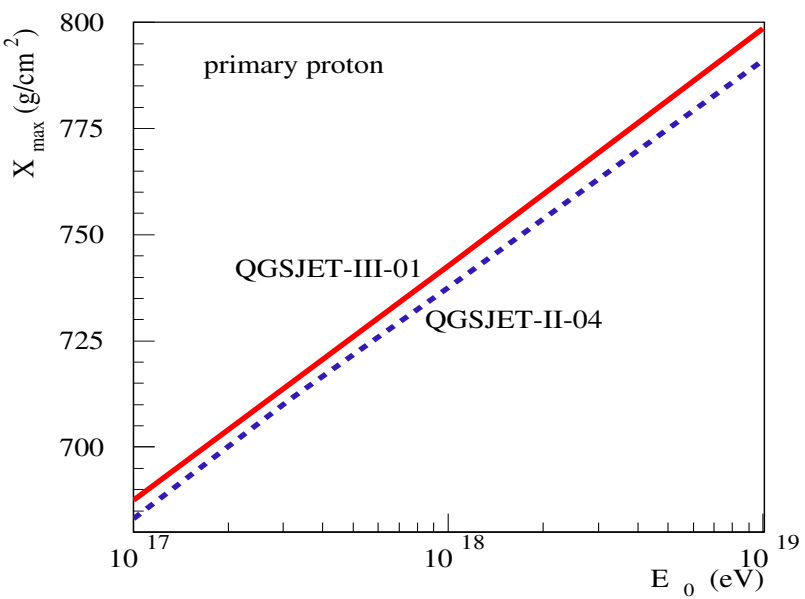

Figure 10. Energy-dependence of the shower maximum depth $X_{\max }$, as calculated using QGSJET-III-01 and QGSJET-II-04 solid and dashed lines respectively.

$(\mathbb{R} \mathbb{R})$ contributions in Monte Carlo generators would be very timely.

The author acknowledges the support by Deutsche Forschungsgemeinschaft (project OS 481/2-1).

\section{References}

[1] N. N. Kalmykov and S. S. Ostapchenko, Phys. Atom. Nucl. 56, 346 (1993).

[2] N. N. Kalmykov, S. S. Ostapchenko and A. I. Pavlov, Bull. Russ. Acad. Sci. Phys. 58, 1966 (1994).

[3] N. N. Kalmykov, S. S. Ostapchenko and A. I. Pavlov, Nucl. Phys. Proc. Suppl. 52B, 17 (1997).

[4] S. Ostapchenko, Nucl. Phys. Proc. Suppl. 151, 143 (2006).

[5] S. Ostapchenko, Phys. Rev. D 74, 014026 (2006).

[6] S. Ostapchenko, Phys. Rev. D 83, 014018 (2011).

[7] S. Ostapchenko, EPJ Web Conf. 52, 02001 (2013).
[8] H.J. Drescher, M. Hladik, S. Ostapchenko and K. Werner, J. Phys. G 25, L91 (1999).

[9] S. Ostapchenko, H. J. Drescher, F. M. Liu, T. Pierog and K. Werner, J. Phys. G 28, 2597 (2002).

[10] S. Ostapchenko, Phys. Lett. B 636, 40 (2006).

[11] S. Ostapchenko, Phys. Rev. D 77, 034009 (2008).

[12] S. Ostapchenko, Phys. Rev. D 81, 114028 (2010).

[13] T. K. Gaisser and F. Halzen, Phys. Rev. Lett. 54, 1754 (1985).

[14] J. C. Collins, D. E. Soper and G. F. Sterman, Nucl. Phys. B 308, 833 (1988).

[15] J. C. Collins, D. E. Soper and G. F. Sterman, Adv. Ser. Direct. High Energy Phys. 5, 1 (1989).

[16] R. Astalos et al., e-Print: arXiv:1506.05829 [hep$\mathrm{ph}$.

[17] B. Blok, Yu. Dokshitzer, L. Frankfurt and M. Strikman, Phys. Rev. D 83, 071501 (2011).

[18] B. Blok, Yu. Dokshitzer, L. Frankfurt and M. Strikman, Eur. Phys. J. C 72, 1963 (2012).

[19] M. Diehl, D. Ostermeier and A. Schafer, JHEP 1203, 089 (2012).

[20] V. A. Abramovskii, V. N. Gribov and O. V. Kancheli, Sov. J. Nucl. Phys. 18, 308 (1974).

[21] G. Antchev et al. (TOTEM Collaboration), Europhys. Lett. 101, 21002 (2013).

[22] G. Antchev et al. (TOTEM Collaboration), Europhys. Lett. 101, 21004 (2013).

[23] G. Antchev et al. (TOTEM Collaboration), Phys. Rev. Lett. 111, 012001 (2013).

[24] G. Antchev et al. (TOTEM Collaboration), e-Print: arXiv:1712.06153 [hep-ex].

[25] G. Aad et al. (ATLAS Collaboration), Nucl. Phys. B 889, 486 (2014).

[26] T. C. Rogers, A. M. Stasto and M. I. Strikman, Phys. Rev. D 77, 114009 (2008).

[27] L. Frankfurt, M. Strikman, D. Treleani and C. Weiss, Phys. Rev. Lett. 101, 202003 (2008). 
[28] G. Antchev et al. (TOTEM Collaboration), Europhys. Lett. 101, 21003 (2013).

[29] G. Antchev et al. (TOTEM Collaboration), Phys. Rev. Lett. 111, 262001 (2013).

[30] T. C. Rogers and M. Strikman, Phys. Rev. D 81, 016013 (2010).

[31] S. Ostapchenko and M. Bleicher, Phys. Rev. D 93, 034015 (2016).

[32] V. Khachatryan et al. (CMS Collaboration), JHEP 01, 079 (2011).

[33] R. L. Jaffe and M. Soldate, Phys. Lett. 105B, 467 (1981).

[34] R. L. Jaffe and M. Soldate, Phys. Rev. D 26, 30 (1982).

[35] R. K. Ellis, W. Furmanski and R. Petronzio, Nucl. Phys. B 207, 1 (1982).

[36] R. K. Ellis, W. Furmanski and R. Petronzio, Nucl. Phys. B 212, 29 (1983).

[37] J.-W. Qiu, Phys. Rev. D 42, 30 (1990).

[38] M. Luo, J.-W. Qiu and G. Sterman, Phys. Rev. D 50, 1951 (1994).
[39] J.-W. Qiu and I. Vitev, Phys. Rev. Lett. 93, 262301 (2004).

[40] J.-W. Qiu and I. Vitev, Phys. Lett. B 632, 507 (2006).

[41] G. Aad et al. (ATLAS Collaboration), Eur. Phys. J. C 72, 1926 (2012)

[42] V. Khachatryan et al. (CMS Collaboration), Phys. Rev. D 92, 012003 (2015).

[43] K. Nakamura et al. (Particle Data Group), J. Phys. G 37, 075021 (2010).

[44] G. Aad et al. (ATLAS Collaboration), New J. Phys. 13, 053033 (2011).

[45] G. Aad et al. (ATLAS Collaboration), Phys. Lett. B 758, 67 (2016)

[46] G. Aad et al. (ATLAS Collaboration), Eur. Phys. J. C 76, 502 (2016).

[47] V. Khachatryan et al. (CMS Collaboration), Phys. Rev. D 92, 112001 (2015).

[48] O. Adriani et al. (LHCf Collaboration), Phys. Lett. B 750, 360 (2015).

[49] O. Adriani et al. (LHCf Collaboration), e-Print: arXiv:1808.09877 [hep-ex] 